\title{
Transitive avoidance games on boards of odd size
}

\author{
Matthias Gehnen \\ RWTH Aachen \\ North Rhine-Westphalia, Germany \\ gehnen@cs.rwth-aachen.de
}

\author{
Eberhard Triesch \\ Lehrstuhl II für Mathematik \\ RWTH Aachen \\ North Rhine-Westphalia, Germany \\ triesch@math2.rwth-aachen.de
}

Submitted: Jan 27, 2021; Accepted: Sep 6, 2021; Published: Nov 19, 2021

(C) The authors. Released under the CC BY-ND license (International 4.0).

\begin{abstract}
Avoidance (or misère) games are a type of positional games. Two players alternately claim points of a set $N$ (the 'board' of the game). The game is determined by a family $L$ of subsets of $N$ and the following rule: The first player who claims every point of some set in $L$ loses the avoidance game.

The game is called transitive if the group of all permutations of $N$ leaving $L$ invariant acts transitively on $N$. Johnson, Leader and Walters show that for a board size which is neither a prime number nor a power of two there are transitive avoidance games where the first player can force his win. For primes of size at least 17, the corresponding problem remained open. We are going to close this gap and prove that for all primes $n$ of size at least 17 there are also transitive avoidance games with board size $n$ where the first player can force his win.
\end{abstract}

Mathematics Subject Classifications: 91A46, 05C57

\section{Introduction}

In combinatorial game theory, positional games are usually defined as follows: There is a finite set which is called the board and a family of subsets from this board, sometimes called lines. Two players alternately claim unoccupied elements (or 'points') from the board. In an achievement game the first player who claims every point of some line wins. In an avoidance game, the opposite rule applies: the first player claiming every point of some line loses. We say that a player wins a game if he has a strategy that guarantees him to win. It is also possible for a game to end in a draw if none of the players has a winning strategy.

This paper deals with the avoidance variant which has also been discussed in [1], [2], $[4],[5]$ and [6]. 
For achievement games, a strategy stealing argument shows that any game ends either with a win for the first player or in a draw. Considering the avoidance variant, it seems that, intuitively, the second player has an advantage. It is however possible to construct avoidance games in which the first player wins: As a trivial example, let $x$ denote some particular point on the board and define the lines as all one element subsets of the board except $\{x\}$. By choosing $x$ in his first move, the first player obviously wins.

The situation becomes more subtle if we insist that our games are transitive: A game is transitive if the automorphism group of the game acts transitively on the points of a board. Here, the automorphism group is the group of all permutations of the board mapping lines to lines. Informally, in a transitive game all points on the board 'look the same' [5].

Johnson et al. [5] investigate the existence of transitive avoidance games depending on the board size $n$. If $n$ is neither a prime number nor a power of two they show that there are such games. They also prove that such games cannot exist if $n$ is a power of two or equal to 3,5 or 7 . If $n$ is 11 or 13 , however, they found transitive avoidance games which are a first player win by computer search.

In this paper, we show that for all primes $n \geqslant 17$, there is a transitive avoidance game which is a first player win thus answering the open question 1 from [5].

The construction works for all odd board sizes $n \geqslant 17$ and is the central result of [3]. We give the details in the next section. Player I and Player II are abbreviated by PI and PII, respectively.

\section{$2 \quad$ A game on odd board sizes $n \geqslant 17$}

Theorem 1. For every odd board size $n \geqslant 17$ there exists a transitive avoidance game that is a PI win.

Proof. Suppose that an odd number $n \geqslant 17$ is given. Our board is the set $\{1, \ldots, n\}$ and we arrange the numbers on a circle in the natural way. So, $i$ is followed by $i+1$, $1 \leqslant i \leqslant n-1$, and $n$ is followed by 1 . Two consecutive positions on the circle are also called adjacent. A set of consecutive points is called a block. Lines are all subsets of the board where

1. the subset includes a block of four elements, or

2. the complement of the subset does not include a block of four elements.

For example, for a board of size seventeen the subset $\{1,2,3,7,8,11,17\}$ forms a line since it includes the block $\{17,1,2,3\}$.

Obviously, the set of lines is invariant under rotations of the circle and thus our game is transitive. There can also be no draw: If all $n$ positions are claimed and PI did not claim a block of length four, then the PII positions form a line since the sets of PI and PII positions are complements of each other. 
If, during the game, one player has claimed $k \geqslant 2$ points, then these points dissect the rest of the board into $k$ blocks. Each such block will be called a gap (for this player). If a position was not claimed by either of the players yet it is called free. A set of positions is free if each of its elements is. It is possible that some of the $k$ blocks are empty.

We now describe the winning strategy for PI. It is divided into five phases.

\section{Strategy Phase I (Start of the game)}

PI begins and claims point 1.

PII claims an arbitrary point.

By claiming his second point, there will be two gaps of PI between his two points. PI now claims the point in such a way that: (1) the point of PII lies in a gap of even size; (2) this gap has at least four points and (3) given (1) and (2), this gap is as small as possible. Since $n$ is odd and at least 17, it follows that the other gap size is odd and at least seven. PII now claims his second point and PI chooses a point in his odd-sized gap as follows:

If the second point of PII lies in the same PI-gap as the first point, then PI claims his third point in such a way that his odd-sized gap will be divided into two gaps of even size at least two. If not, PI proceeds similarly but ensures in addition that the second point of PII is in an (even-sized) PI-gap of size at least 4 . We denote by $S$ the set of the first three positions claimed by PI.

Now, every gap between the three points from $S$ is of even size at least two; if a point of PII is in a gap then its size is at least four. We now partition all three gaps into pairs of two adjacent points (blocks of length 2) and denote this set of pairs by $A$. Depending on the situation after the previous moves, we proceed as follows (see Figure 1):

1. Both points of PII are in the same or in two adjacent pairs from $A$ (in the same gap). Then, it is possible to consider two adjacent pairs as a block $B$ of four points with two free points and two points of PII. Continue with Phase IV of the strategy.

2. One gap contains three consecutive pairs $a, b, c$ (in this order) from $A$ such that $a$ and $c$ contain the two points claimed by PII and $b$ is free. We denote by $C$ and $D$ the two blocks consisting of the pairs $a, b$ and $b, c$, respectively. Continue with Phase III of the strategy.

3. For every other case: Consider two disjoint blocks $C$ and $D$, each consisting of two adjacent pairs from $A$ and each containing one of the points claimed by PII. Continue with Phase II of the strategy.

\section{Strategy Phase II (Two disjoint blocks of length four)}

The board is partitioned as follows: We have the set $S$ and two disjoint 4-blocks $C$ and $D$ with one PII-point in each block. The other positions in $C$ and $D$ are free. The remaining points are partitioned by pairs from $A$. The pairs can be free or they are mixed, (so containing both, one PI- and one PII-position). 


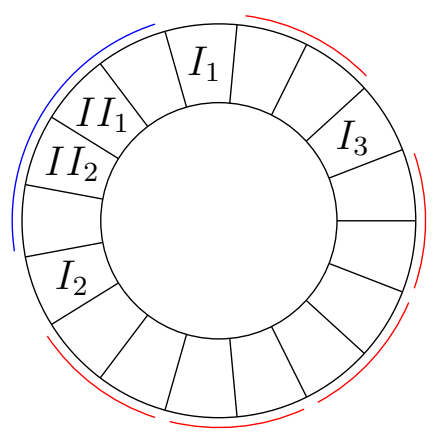

Case 1 in Phase I

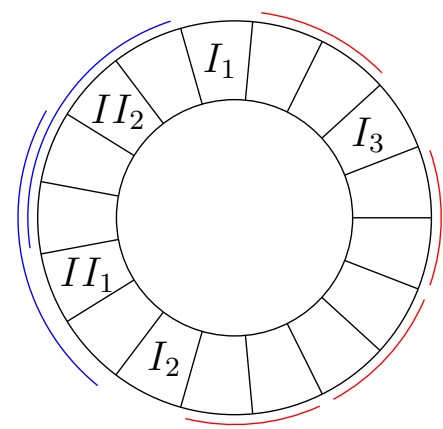

Case 2 in Phase I

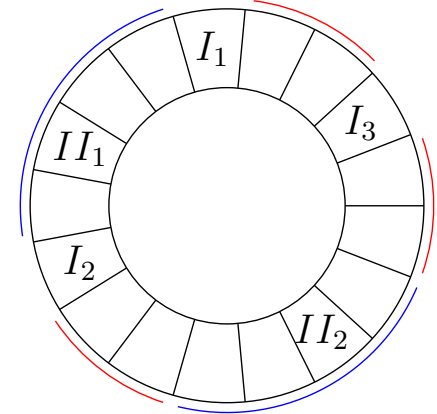

Case 3 in Phase I

Figure 1: Possible board after Phase I with a board of size seventeen. PI claims $I_{1}, I_{2}$, and $I_{3}$ in this order, PII claims $I I_{1}$ and $I I_{2}$. The pairs are marked red, the blocks of four blue.

If PII claims a point of a free pair disjoint from $C$ and $D$, then PI claims the other point of that pair. Stay in Phase II of the strategy.

If PII claims a free point in $C$ or $D$, then we rename this block as $B$ and break up the other block into two pairs $a$ and $b$ from $A$. One of the pairs, say $a$, contains a PII-position. Now PI claims the free position in $a$. We leave Phase II and go to Phase IV.

\section{Strategy Phase III (Two blocks of length four with overlap)}

The board is partitioned in $S$, in two blocks $C$ and $D$ consisting of the pairs $a, b$ and $b, c$ as described above and in pairs from $A$ which are either free or mixed.

If PII claims a point of a free pair disjoint from $C$ and $D$, then PI claims the other point of that pair. Remain in phase III of the strategy. If PII claims a free point in $a$ or $b$, we rename $C$ as $B$. PI claims the free position in $c$ and we go to phase IV. Similarly, if PII claims the free position in $c$, we rename $D$ as $B$. PI claims the free position in $a$ and we go to Phase IV.

\section{Strategy Phase IV (One block of length four with two points of PII)}

The board is partitioned in $S$, in the block $B$ with two PII-points and two free points, as well as in pairs from $A$. If PII claims a point of a free pair disjoint from $C$ and $D, \mathrm{PI}$ claims the other point of that pair. Remain in Phase III of the strategy.

If PII claims a point of a free pair disjoint from $B$, then PI claims the other point of that pair. Stay in Phase IV of the strategy.

If PII claims a point from $B$ and there are free pairs left, then PI claims a point of a free pair. Go to Phase V of the strategy.

If PII claims a point from $B$ and there are no free pairs left, then the free point in $B$ is the only free point left on the board. We show that PII has lost and therefore PI do not have to react. To see this, note that, for all pairs in $a \in A$, the following holds: 
If $a$ contains a PI-position, then the other position in $a$ was claimed by PII. It follows that PI-blocks can only have sizes one, two or three, where the case of length three is only possible if the central element is from $S$. Since PI never claimed a position in $B$, we see that PI never completed a line. Suppose that there is a block of length four without PII-positions. Since this block has to contain three PI-positions and the free point from $B$. By our observation on the structure of PI-blocks of length three, the pair $a \in A$ containing the free position must also contain a PI-position. Since $a$ has to be contained in $B$, we have a contradiction.

\section{Strategy Phase V (One block with three points of PII)}

The board is partitioned in $S$, in the block $B$ with one free point and three PII-points, as well as possibly three kinds of pairs from $A$ : Free pairs, mixed pairs and exactly one pair with one PI- and one free position.

If PII claims the last point in the block of four, then he obviously completed the line $B$.

If PII claims a point of a free pair, PI claims the other point of the pair. Stay in Phase $\mathrm{V}$ of the strategy.

If PII claims the free point in the pair which already includes one point of PI and there is a free pair left, then PI claims a point in a free pair. Note that this also not complete a line by PI. Stay in Phase V.

If there is no free pair left, then the only free point is in $B$. This situation is very similar to the case discussed at the end of Phase IV.

Summarizing, we have shown that by following the strategy described above, PI has not completed a line after a total of $n-1$ moves whereas PII has. The $n$th point in a game of odd board size must be set by PI, but since PII has lost before the game would have stopped before playing the $n$th point. It follows that PI wins.

Corollary 2. A transitive avoidance game that is a PI win exists for all board sizes except three, five, seven and the powers of two.

\section{Remarks}

First we would like to thank our anonymous referees for the comments that helped to improve the presentation of the game. We will conclude with two remarks prompted by their comments:

We were asked to give an intuition on how to get an idea for such a game: The constructions for avoidance games in [5] are using integer factorization for the design of the board. Since we cannot use this to construct the automorphism group for board sizes that are primes, we have chosen the rotation as a very simple and natural group.

Then with the fixed board we started with a simple rule like forbidding three consecutive points. While trying to find a strategy for PI, we found reasons why we could not find a winning strategy for PI. Then you can adapt the reasons to try to construct a 
game that fulfills the wanted conditions. After some iterations it is possible to get some intuition for the setting and to finally find the resulting game.

Second, it was hinted that it is possible to modify the first phase, such that the game also works for board sizes of 13 and 15. In particular, this allows us to give a constructive game for size 13 .

This can be achieved by claiming the second item of PI in a way such that there is a free gap of size two between the two points of PI. If the second point of PII is in the gap of size two, then PI can claim the other point of this gap. However, if PII has claimed a second point in the big gap (with size of 9 or 11), then PI is able to claim a point in this gap in such a way that the board fulfills the condition of Phase $I I, I I I$ or $I V$.

\section{References}

[1] J. Beck, Ramsey Games, Discrete Math., 249(1-3):3-30, 2002.

[2] J. Beck, Combinatorial games, Encyclopedia of Mathematics and its Applications 114, Cambridge University Press, Cambridge, 2008.

[3] M. Gehnen, Vermeidungsspiele, Master's thesis, RWTH Aachen, 2020.

[4] F. Harary, Achievement and avoidance games for graphs, Graph theory (Cambridge, 1981), Ann. Discrete Math. 13:111-119. North-Holland, Amsterdam-New York, 1982.

[5] J. R. Johnson, I. Leader and M. Walters, Transitive Avoidance Games, Electron. J. Comb., 24(1):\#P1.61, 2017.

[6] G. Simmons, The game of SIM, J. Rec. Math., 2(2):66, 1969. 